\title{
IMMUNOLOCALIZATION OF PHOSPHODIESTERASE ISOENZYMES IN RAT TISSUES USING CONFOCAL MICROSCOPY
}

\author{
Beverly E. Maleeff, Rosanna C. Mirabile, Timothy K. Hart, Heath C. Thomas, Lester W. Schwartz
} and Stephen J. Newsholme

Department of Safety Assessment, GlaxoSmithKline, King of Prussia, PA

Cyclic nucleotide phosphodiesterases (PDE) are a family of structurally related enzymes responsible for degrading cAMP and cGMP in eukaryotic cells ${ }^{[1]}$. Many tissues express members of multiple PDE families ${ }^{[2]}$. PDE type 3 (PDE3) has two known isoforms and has been purified from a number of tissues ${ }^{[3]}$. One of its known functions is relaxation of vascular smooth muscle. PDE type 4 (PDE4) has four known isoforms, has been purified from tissues including germ cells and vascular smooth muscle, and is believed to play a role in blocking contraction of airway smooth muscle ${ }^{[4]}$. PDE4 inhibitors are among several drug classes that induce segmental medial necrosis of mesenteric arteries after administration to rats. The nature and distribution of the lesions suggest a consequence of extreme localized disturbance of smooth muscle tone. We hypothesized that regional distribution of PDE3 and PDE4 isoenzymes predisposes the rat mesenteric artery to damage; therefore, we explored the distribution of these isoenzymes in a panel of tissues that may be implicated in these drug interactions.

Mesenteric, femoral and carotid arteries, aorta, liver and lung were collected from untreated male Sprague-Dawley rats. Frozen transverse sections were prepared and fixed in ethanol:acetone, blocked with BSA, incubated with primary antibody $(\mathrm{Ab})$ (rabbit anti-rat PDE3B; rabbit anti-rat PDE4A, B, or C; mouse anti-rat PDE4D), followed by secondary antibodies conjugated to the fluorochrome Cy5, and counterstained with DAPI (fluorescent nuclear stain). Whole mounts of mesenteric arteries, briefly fixed with formaldehyde, were stained as above and enrobed in agar. All tissues were examined with a Zeiss LSM-510 confocal laser scanning microscope using $488 \mathrm{~nm}$ and $568 \mathrm{~nm}$ excitation lines of an $\mathrm{Ar} / \mathrm{Kr}$ laser, the $633 \mathrm{~nm}$ excitation line of a HeNe laser and appropriate emission filters, or in differential interference contrast (DIC) mode.

In vascular sections all PDE3 and PDE4 isoenzymes were localized, to varying degrees, throughout the medial smooth muscle layer and within the endothelium. In whole vessel mounts, PDE3B isoenzymes were found only on the outer surface associated with adventitial adipocytes, whereas all 4 PDE4 isoenzymes were localized diffusely throughout the medial smooth muscle; in all cases, there was no evidence of segmental distribution along the vascular wall. In sections of liver and lung, PDE4 isoenzymes were localized to varying degrees in hepatic arteries and alveolar capillaries.

The principal PDE4 isoenzymes (A, B, C and D) are expressed in medial smooth muscle and endothelium of rat mesenteric arteries and therefore could be involved in mediation of altered tone, either dependent upon or independent of endothelial activity. Diffuse expression of PDE4 isoenzymes along the vascular wall suggests that the segmental lesion induced by PDE4 inhibitors is unlikely related to local differences in PDE4 enzyme distribution. Similar findings with PDE3B expression support the rejection of our hypothesis. Therefore, there is still no clearcut mechanism for the segmental nature of damage occurring in rat mesenteric vasculature following exposure to PDE4 inhibitors. 
References

1. Müller T, Engels P and Fozard JR. Trends in Pharmacol. Sci. 17(8): 294-298, 1996.

2. Liu H, and Maurice DH. British J. Pharmacol. 125(7): 1501-1510, 1998.

3. Degerman E, Belfrage P and Manganiello, VC. J. Biol. Chem. 272(11): 6823-6826, 1997.

4. Saldou N, et al. Cellular Signaling 10(6): 427-440, 1998.
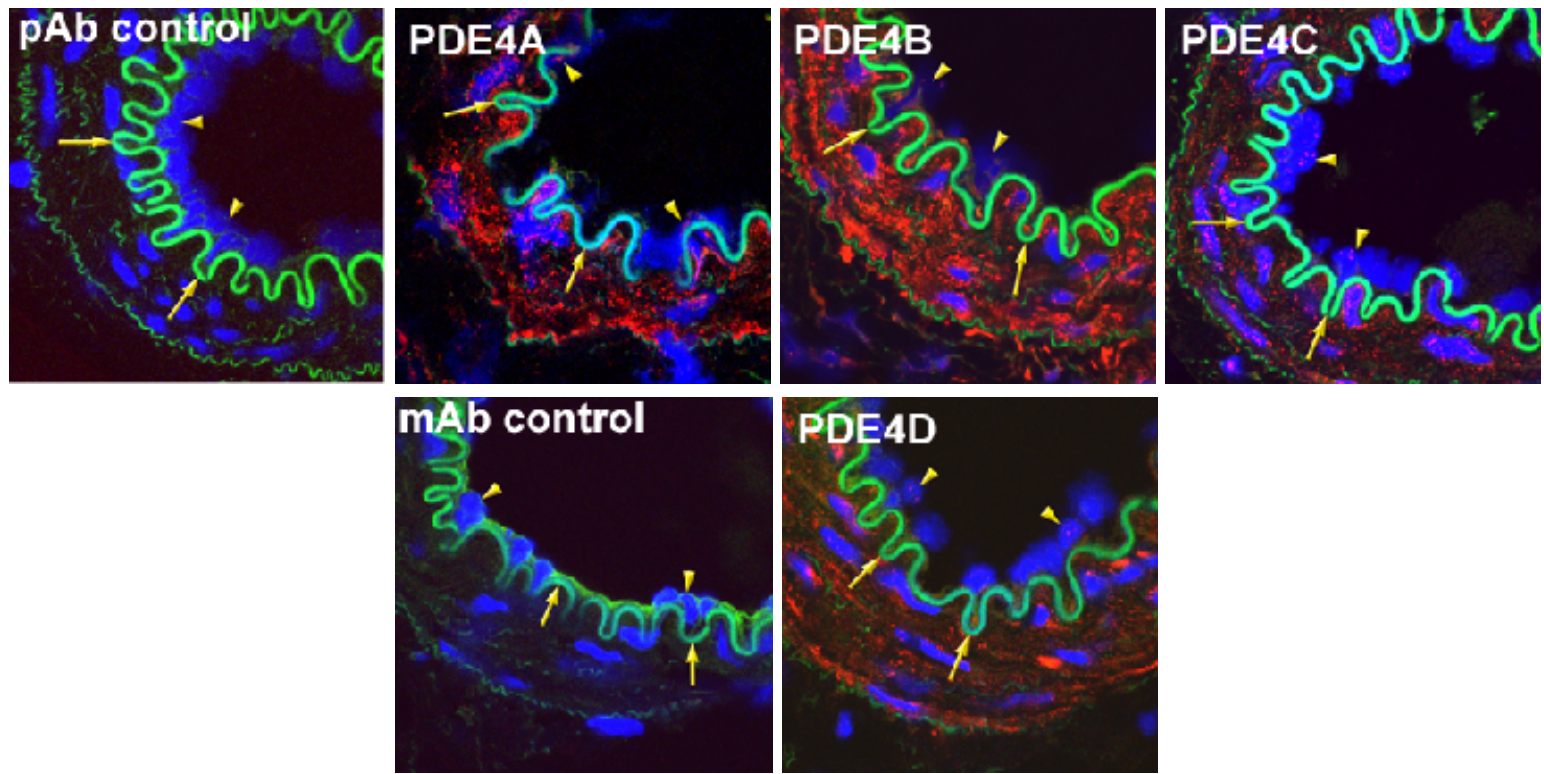

Figure 1. Transverse sections of mesenteric arteries stained with polyclonal Abs PDE4A-C or monoclonal Ab PDE4D, with negative controls. Elastin in the internal elastic lamina (arrows) is autofluorescent. Endothelial cells line the lumen of each vessel (arrowheads). Original magnification 100x.
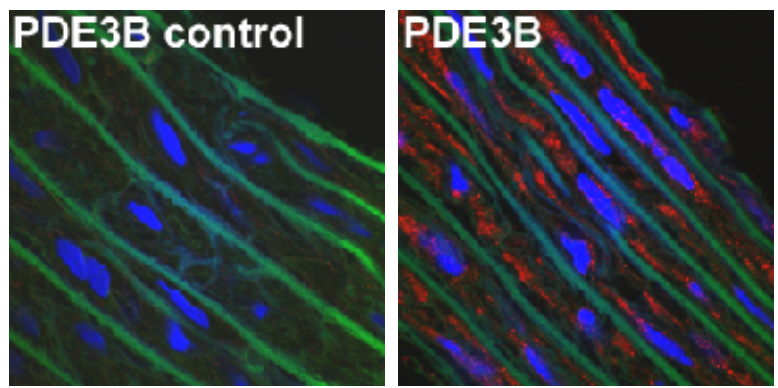

Figure 2. Transverse sections of aorta stained with anti-PDE3B Ab. Original magnification 100x.
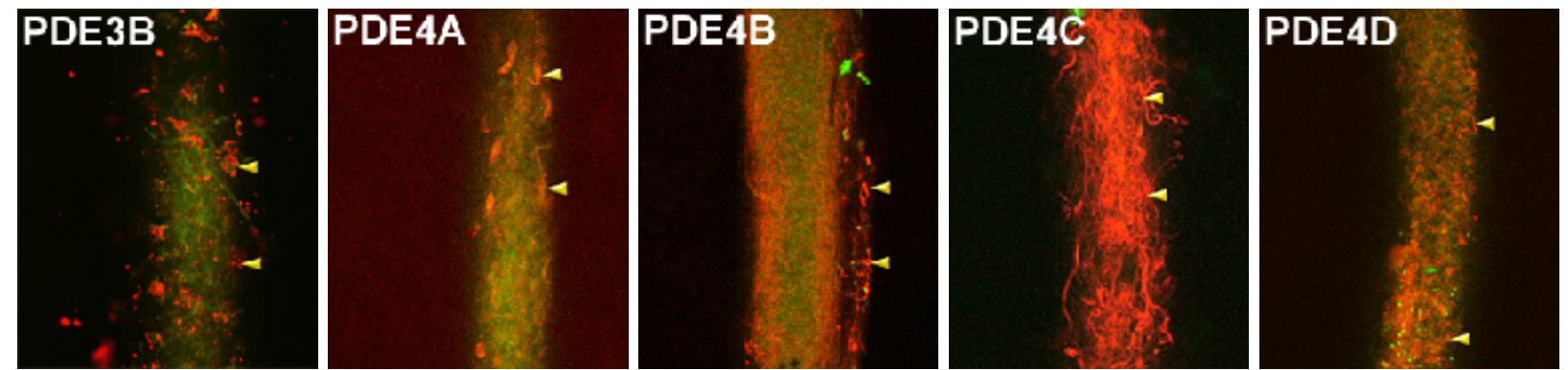

Figure 3. Adventitial surface of whole-mount mesenteric arteries, stained for PDE3B and PDE4A-D. Note the stained surface structures (arrowheads). Original magnification 40x. 